\title{
Ampliación del ciudadano desde un enfoque sistémico en Pereira (Colombia) y Santiago de Chile (Chile)*
}

\author{
Expansion of the citizen, from a systemic approach in \\ Pereira (Colombia) and Santiago de Chile (Chile)
}

\author{
Joaquín Andrés Gallego Marín** \\ César Augusto Castaño*** \\ Erika María Bedoya $* * * *$ \\ Artículo de reflexión \\ Fecha de Recepción: 30 de noviembre de 2020 \\ Fecha de Aceptación: 22 de enero de 2021
}

\section{Para citar este artículo:}

Gallego Marín, J. A., Castaño, C. A. y Bedoya, E. M. (2021). Ampliación del ciudadano desde un enfoque sistémico en Pereira (Colombia) y Santiago de Chile (Chile). Revista Análisis Jurídico-Político, 3(5), 111-141. https://doi.org/10.22490/26655489.4366

\footnotetext{
* El presente artículo hace parte del proyecto Incidencia de las organizaciones de la sociedad civil (osc) en la formulación y ejecución de la política en la ciudad de Pereira 20122016, el cual fue financiado con recursos de la convocatoria n. ${ }^{\circ} 6$ de 2017 de la Universidad Libre, sede Pereira.

** Abogado, licenciado en Etnoeducación y Desarrollo Comunitario, magíster en Educación y candidato a doctor en Derecho. Docente investigador y titular en la Universidad Libre, sede Pereira. Correo electrónico: joaquina.gallegom@unilibre.edu.co; jgallegomarin@gmail.com ORCID: https://orcid.org/0000-0001-6196-7352

*** Licenciado en Etnoeducación y Desarrollo Comunitario, magíster en Comunicación Educativa y estudiante en el Doctorado en Estudios Territoriales, Universidad de Caldas. Docente investigador de la Universidad Libre, sede Pereira. Correo electrónico: cesara.castanog@unilibre.edu.co. oRCID: https://orcid.org/0000-0002-0160-0302

**** Licenciada en Etnoeducación y Desarrollo Comunitario, magíster en Comunicación Educativa, suficiencia investigadora en el Doctorado en Paz Conflicto y Democracia, y candidata en el Doctorado Interinstitucional en Educación. Docente investigadora de la Universidad Libre, sede Pereira. Correo electrónico: erikam.bedoyah@unilibre.edu.co ORCID: https://orcid.org/0000-0003-3228-3087
} 


\section{Resumen}

Este artículo de reflexión plantea el concepto de ampliación de ciudadanía. Su pretensión se enfoca en dar respuesta a la pregunta: ¿cómo se amplía el sistema ciudadano en su relación con la administración pública, a partir del referente decisión en contextos territoriales de las ciudades de Pereira (Colombia) y Santiago de Chile (Chile)? El lector encontrará cuatro aspectos de discusión de orden teórico-metodológico y resultados: 1) el ciudadano no en la sociedad, sino con la sociedad; 2) el sistema político y el ciudadano como sistema psíquico; 3) las formas de la relación administración pública/público ciudadanos en el escenario de las ciudades de Santiago de Chile (Chile) y Pereira (Colombia); y 4) ampliación/ no aplicación del ciudadano, como un problema fundado en la exclusión/inclusión, a manera de conclusiones.

Palabras clave: ciudadano, administración pública, sistemas sociales, decisión.

\section{Abstract}

This article for reflection raises the concept of expanding citizenship. His claim is focused on answering the question: how does the citizen system expand in its relationship with the public administration, based on the decision reference in territorial contexts of the cities of Pereira (Colombia) and Santiago de Chile (Chile)? The reader will find four theoretical-methodological discussion aspects and results: 1) the citizen not in society, but with society; 2 ) the political system and the citizen as a psychic system; 3) the forms of the relationship between public administration/public citizens in the scenario of the cities of Santiago de Chile (Chile) and Pereira (Colombia); and 4) extension/non-application of the citizen, as a problem based on extension/non-application, by way of conclusions.

Keyworks: citizen, public administration, social systems, decision. 


\section{INTRODUCCIÓN}

La deliberación sobre el concepto de ampliación del ciudadano es una reflexión contemporánea, que no solo atañe a las democracias latinoamericanas, sino que se extiende a la consideración mundial en el marco de los sistemas políticos, económicos y sociales, los cuales se hacen evidentes en los diferentes movimientos de indignados o de organizaciones de la sociedad civil, con ánimo de incidir en la decisión de los asuntos públicos.

Este artículo orienta la discusión hacia la manera como se amplía el sistema ciudadano en su relación con la administración pública, a partir del referente decisión en contextos territoriales de las ciudades de Pereira (Colombia) y Santiago de Chile (Chile). Dicho diálogo se enlaza desde la revisión y actualización de la investigación titulada Incidencia de las organizaciones de la sociedad civil (OSC) en la formulación e implementación de la política pública en la ciudad de Pereira 2012-2016, la cual finalizó en el 2019. En el presente artículo se amplía el área problémica de la ciudadanía en contextos reales.

El anclaje sobre la observación del ciudadano se direcciona mediante su relación con la administración pública, como dos sistemas autónomos enlazados de manera comunicativa mediante el elemento participación ciudadana en un doble contexto: Pereira (Colombia) y Santiago de Chile (Chile). Se propone brindar una aproximación comprensiva de la construcción de ciudadanía mediante la relación funcional: ciudadano/administración pública.

Para establecer la forma de ampliación del ciudadano mediante dicha relación, es importante (metodológicamente) observar y exponer cómo se efectúan los intercambios de relación entre el ciudadano y la administración pública. Lo anterior, a partir de la teoría de los sistemas sociales del sociólogo alemán Niklas Luhmann, que se ubica en el pensamiento relacional como medio observacional de la sociedad formada por sistemas sociales hilados a través de la comunicación. De allí, se propone observar la sociedad mediante el referente de los sistemas sociales en relación autorreferente con la 
heterorreferencia de los demás sistemas, como los sistemas psíquicos y sus propios sistemas o subsistemas.

A partir de allí, se da la edificación teórica desde el principio de lo complejo, en la forma de la sociedad descentrada y polivalente, donde no es posible considerar un centro radicalizado de manera estructural. El avance es observar la sociedad en su diferenciación funcional radicalizada en los diferentes sistemas conforme a sus propias funciones y cómo estos se enlazan de manera comunicativa mediante la forma sistema/entorno como complemento.

Se busca, entonces, observar al ciudadano y a la administración pública como dos sistemas autorreferentes que se entrelazan como un alter ego, hilados en torno a las acciones políticas decisivas en un contexto territorial. Esta perspectiva de visión funcionalista tiende a tener presente la distinción, selección y decisión en cada sistema, que se enlazan bajo el código central del sistema político; pese a que, ni el ciudadano ni la administración pública estén orientados bajo unas prescripciones políticas de forma diferente.

Una vez supuestos estos referentes desde la visión teórica, dejan ver cómo el ciudadano de Santiago de Chile (Chile) y el de Pereira (Colombia) desarrollan dinámicas de distinción, selección y decisión ante el sistema de la administración pública, mediante la activación de mecanismos especializados en su propia función. A esto nos referimos como mecanismo de participación. Todo lo queallíse presente se aprehenderá bajo la apreciación de relación entre dos sistemas vinculados mediante el material de comunicación orientado por la distinción: poder/no poder y gobierno/oposición, con las que se desarrolla la sensibilidad y la capacidad de procesamiento de información en el contexto de observación ya indicado.

\section{El ciudadano no en la SOCIEDAD, Sino CON LA SOCIEDAD}

"La sociedad es compleja, cambiante y también impredecible". Con esta afirmación Santiago Montenegro (2009) cierra la columna 
titulada ¿Sociedad estática o sociedad dinámica? publicada en el diario el Espectador de Colombia. La opinión del columnista parte de la reflexión de la sociedad conforme a la postura heraclitiana sobre el constante cambio al que está sometida. Por ello, el columnista muestra tres características de la sociedad colombiana:

Primero, una sociedad como la nuestra es compleja y está formada por una multiplicidad de actores políticos, regionales, sectoriales y de otras características. Segundo, dicha sociedad es dinámica, cambiante y en muchos aspectos impredecible. Tercero, en esa sociedad compleja, diversa y cambiante, la solución de unos problemas crea, casi que automáticamente, otros problemas. (párrafo 2)

Estas selecciones y distinciones del columnista nos presentan una sociedad compleja conforme a las formas de observarla, seleccionarla e indicarla. Lo anterior, da una posibilidad de considerar la sociedad más allá de su operación racional explicativa:

- Primero, determinar a la sociedad como objeto de estudio y precisar el sujeto dentro de esta. La evolución sobre el sujeto ha estado enmarcada por la búsqueda de su libertad, construyéndose una representación desde el sujeto opuesta a la del objeto. Por ejemplo, los planteamientos de Platón, Aristóteles, Zenón de Citio, Descartes, Kant, o en la actualidad, con Husserl o Heidegger, en los que el sujeto tiene la capacidad de conocer y dominar lo externo a sí mismo, al mundo o la sociedad; es decir, es aparte, distante de ellos. Lo que tiene del mundo es diferente al sujeto.

- Segundo, la oposición irreconciliable entre sujeto y objeto. Esta oposición ha influido constantemente los estudios sociales. Muestra de ello los presupuestos analíticos de la sociedad planteados por Emile Durkheim (1982), quien ha creado un orden holístico en la concepción de sociedad como una unidad en la que los individuos están agregados. De otro lado, Max Weber o Georg Simmel (2002) se enfocaron sobre el in- 
dividuo y su aportación a la sociedad. Esta última rescata la realidad social mediante los procesos de acción recíproca, "que para el individuo significa el hecho de estar socializado" (Simmel, 2002, p. 82). Este intento, desde la razón del observador de la sociedad, ha puesto en una exploración donde el individuo y la sociedad se comportan como dos formas independientes y sin relación, que responden a la condición del $a$ priori kantiano.

- Tercero, la sociedad como algo seguro. La cultura griega ha dejado un legado inconmensurable, un pensamiento racionalista como medio orientador de la vida social. Sin embargo, la evolución cultural de las formas de vida en la modernidad muestra que la sociedad ha tenido procesos de trasformación que amenazan la estabilidad del referente social. Como plantea Ulrich Beck (1998), "La consecuencia central es que en la modernidad avanzada la sociedad con todos sus sistemas parciales (economía, política, familia, cultura) ya no se puede comprender de una manera 'autónoma respecto de la naturaleza'" (p. 90). Con esto, el autor esboza la necesaria forma de observar los efectos que producen las condiciones naturales de la vida -individualización-y sus influjos en los sistemas sociales que afectan a las estructuras sociales sembradas en Occidente.

Con Beck, está el arrojo de observar y pensar la sociedad mediante una visión compleja y todas aquellas situaciones, acontecimientos, comunicaciones, relaciones, condiciones, identidades, agentes, roles, lugares, temporalidades, instituciones, acciones y conocimientos de la sociedad para evolucionarlos como ésta lo ha hecho. Este pensamiento permite instituir una comprensión de la sociedad moderna, cuyos contactos sociales se delimitan y mantienen 
mediante operaciones comunicativas ${ }^{1}$, y su base también contempla el principio relacional implicada por la teoría de los sistemas sociales de Niklas Luhmann.

A partir de allí, implica separar de la concepción de unidad de partes conformantes de la sociedad y pasar a la de sistemas diferenciados por funciones específicas de identidad autónoma, pero con la capacidad de referenciarse de otros sistemas. De acuerdo con lo anterior, el derecho, la política, la religión, la familia, la economía, la ciencia, las organizaciones, el arte, la pedagogía y todo aquel sistema poseedor de su propia capacidad de referencia adquiere la capacidad de diferenciación. No obstante, la configuración teórica también trae consigo - mediante la distinción- la ineludible formación del sistema a través de lo recalcado como entorno, pues con este todo sistema adquiere su característica constitutiva como sistema: la demarcación de la visión teórica, la distinción sistema/ entorno.

El planteamiento teórico de los sistemas sociales nos ubica en la mirada de la sociedad a un nivel de relaciones sistémicas entre sus sistemas, subsistemas y sistemas parciales, en donde se repite la distinción sistema/entorno. Esto es una mirada interna de los sistemas sociales, con la que se elaboraría un concepto de sociedad, construida desde los mismos sistemas sociales. Sin embargo, en la

1 Desde la apuesta de Luhmann, este concepto posee una carga semántica densa, la cual proponemos desenvolver de la siguiente manera. Primero, como operaciones desarrolladas por los sistemas sociales y psíquicos, y con el cual éstos se identifican como sistemas autorreferentes. Segundo, las operaciones comunicativas se convierten en el proceso elemental con el que se afianzan los sistemas sociales y, en consecuencia, la sociedad. Así lo podemos entender con Torres (2009), quien indica que "[...] siempre que existe comunicación se forma sociedad, así como al revés: la comunicación no puede acontecer nunca como acontecimiento solitario, sino solo anticipado y reproducido en forma recursiva otras comunicaciones, por tanto, solo en sociedad" (p. 101). Y, tercero, la operación por comunicación necesariamente implica hilar el concepto de sentido para dar cuenta de que los sistemas sociales, los subsistemas y los sistemas psíquicos poseen la capacidad de distinguir y seleccionar ante la información emitida únicamente si se logra una comprensión de lo informado y emitido.

El sentido lo dan las selecciones producidas por las distinciones efectuadas por el sistema ante el acontecimiento emergente que se produce por la relación con el otro sistema y, por ende, es un medio que actualiza las posibilidades del observador del sistema, y con el cual el observador genera diferencias de lo que se percibe como actual y lo que es posible con lo expresado y aprehendido, que lo remite a otra potencialidad. 
teoría de Luhmann se establece otro tipo de sistemas constructores de referenciación, autónomos y con identidad, por ser diferenciaciones funcionales totalmente independientes de la sociedad. Luhmann (1998) los denomina sistemas psíquicos.

Como indica Luhmann (1998), estos sistemas son "[...] aquellos que reproducen conciencia mediante conciencia" (p. 237) o de "vida consciente" (p. 242). Son considerados autónomos, productores de sentido y fundamentados en su propia conciencia. Como resultado, en el entramado de relaciones comunicativas producidas por los sistemas sociales también se produce la de estos con los sistemas psíquicos, integrando de nuevo la forma sistema/entorno.

Este cambio en las formas de la relación, encierran una disposición evolutiva de la sociedad. Esta se forma por sus propias reproducciones; los sistemas psíquicos - como sistemas de conciencia- también elaboran sus reproducciones, que provienen de los pensamientos producidos por los seres humanos. Esto, necesariamente lleva a establecer el cambio de perspectiva de la sociedad, donde los seres humanos no están vinculados en ella por ser sistemas de conciencia autónoma, entornos de la sociedad.

Luhmann pretende superar la condición mostrada por la epistemología clásica de los sujetos inmersos en la sociedad, esquematizados con la relación analítica de sujeto/objeto. Además del llamamiento hecho por el autor acerca de la sociedad, también advierte sobre el carácter reservado de lo humano como una zona de unidad de todo lo social, pues se hallaría una desintegración formada por cada uno de los sistemas sociales, convirtiéndolo de acuerdo con las intenciones, borrando la capacidad de conciencia reflexiva que posee.

Continuando con Luhmann, para resolver el problema de la oposición sujeto/objeto a la forma del sistema psíquico es necesario superar la discusión filosófica de individuo y persona. Esto, con el propósito de trascender al sistema psíquico, que opera de forma autónoma, consiente y productor de sentido. La forma de explicar la independencia del sistema psíquico se vuelve más fácil cuando Luhmann indica que estos "[...] pertenecen al entorno de los sistemas sociales" (1998, p. 236). Esto es, el estudio de la 
sociedad implica poseer una comprensión primigenia de que los seres humanos no están integrados en la sociedad, son un conjunto complejo independiente de esta.

En este punto — de cambio de la concepción dicotómica de sujeto/objeto a sistemas psíquicos bajo la idea de operaciones- se hayan posibles delimitaciones entre este sistema y los sistemas sociales. Por un lado, los sistemas psíquicos se unen comunicativamente con los sistemas sociales $\mathrm{y}$, por lo tanto, establecen una continuidad de operación con estos, pero de forma autorreferente. Esta delimitación es profunda y está cargada de un necesario cambio en la mirada del individuo constituido física, biológica, psicológica y conceptualmente, al de un sistema psíquico en donde se integran distinciones como: individuo/no individuo, persona/no persona, humano/no humano. Dichas distinciones son también las formas de las operaciones del sistema psíquico.

Tanto los sistemas psíquicos como los sociales tienen elementos en común, como la autorreferencia. Sin embargo, dentro de los sistemas psíquicos, esta considera la individualidad como medio de limitación en ese proceso constante de referirse a sí mismo y como una de sus tantas operaciones, como la determinación operativa por la diferencia y la limitación. Como destaca Luhmann (1998), "Mediante la diferencia y la limitación, la conciencia se obliga a sí mismo a tener en cuenta al entorno" (p. 244). Así las cosas, el autor convierte a estas dos determinaciones operativas de la individualidad en imprescindibles para la autorreferencia de los sistemas psíquicos y su distinción de los sistemas sociales.

Pero al mismo tiempo, al hablar de ser humano no se puede reducir a la circunstancia de individuo o persona, pues es la unidad de la distinción entre el sistema psíquico y el biológico. Como nos muestra Luhmann: “[...] hemos optados por la expresión 'ser humano' para asegurar que se trata tanto del sistema psíquico como del sistema orgánico del hombre" (1998, p. 199). Lo que pretende el autor no es descomponer ulteriormente al ser humano, sino respetar la condición integral de este en su relación con los sistemas sociales y, de esta forma, distinguir que el ser humano no se hace a la medida de la sociedad, pero sí se relaciona comunicativamente con ella. 
En consecuencia, la observación de la sociedad funcionalmente diferenciada, los sistemas sociales y el sistema psíquico adquieren sentido cuando — de manera operativa - se relacionan uno con otro; uno como sistema y el otro como entorno. Este planteamiento supone que el entorno se edifica cuando el contexto de los sistemas sociales es reconocido por las operaciones de relación comunicativa que se edifican entre el sistema $X$ en relación con el otro sistema $Y$ (que se constituye como el entorno del $\mathrm{X}$ ), los que, en sus procesos operativos de comunicación, se incluyen o se excluyen uno a otro.

Con esta perspectiva se está creando una ampliación de sentido ante el ciudadano cuando es tratado como sistema psíquico en el contexto de la sociedad moderna. Al ciudadano necesariamente hay que observarlo y tratarlo como ser humano, como sistema. No obstante, como muestran Bedoya et ál. (2004) y Zapata-Barrero (2001), en torno al ciudadano se ha constituido una complejidad operativa por la forma de inclusión/exclusión creada por el sistema político, social, jurídico y económico, en la esfera pública y al ser sometido a las lógicas de referencia de aquellos. De esta forma, al ciudadano se le carga de una complejidad de sentido y su reconocimiento como persona de actuación política queda enredada ante el interés y reclamo de otros sistemas.

De esta manera, la pretensión de autoconciencia e igualdad del ciudadano queda relegada a las organizaciones, el Estado, los políticos o la administración pública, que les reclaman sus obligaciones políticas conforme a medios jurídicos como la Constitución política, las leyes, los decretos, los acuerdos, los reglamentos, los actos administrativos, entre otros. Esto con el propósito de crear exigencias de presentación, las que finalmente terminan estableciendo la dualidad inclusión/exclusión política. De acuerdo con Luhmann (1993):

[...] el ciudadano del bienestar solo toma en consideración sus derechos (que, sin embargo, pueden ser condicionados con la ayuda de la burocracia), y todo el complemento obligatorio sujeto a obligaciones debe servirse a la obligatoriedad de asumir el papel de miembro (de pago) de organizaciones. (p. 111) 
El molde, la referencia o el rol sobre el ciudadano finalmente termina por reducir al sistema psíquico ante una pretensión cerrada de los demás sistemas, como, por ejemplo, el político, que no necesariamente le ayuda a potencializarse como ser humano. Por ello, establecemos el punto observacional del ciudadano, no en su reducción, sino como unidad llamada sistema psíquico autorreferente en relación con otro sistema autorreferente, como, por ejemplo, la política. Así, uno y otro se enlazan como un sistema de conciencia autorreferente conexo con la referencia a los otros, la heterorreferencia.

Las consecuencias de esta posición nos permiten una observación consiente de todo contacto social entre el ciudadano y el sistema político. Esto como una comprensión bajo la forma sistema/ entorno, que encierra el sentido paradójico de los contactos sociales con que lo niega. Es decir, la producción de comunicación que lo lleva a un valor en donde se esconde la opacidad y la capacidad de negar al entorno.

\section{El Sistema Político y El CIUDAdANO COMO SISTEMA PSÍQUICo}

La teoría de los sistemas sociales impulsa al observador de la sociedad a admitir que esta no se puede deducir por principios, normas o eventos de ocurrencia y que la política no se puede interpretar por un marco de conceptos o categorías. Luhmann (1998) destaca la necesidad de comprender el papel relevante que tendría cada sistema dentro de la sociedad, al ser conscientes de que cada sistema cumple una función específica dentro de la trama de relaciones y contribuyen a la construcción de la referencia de los otros sistemas.

Con esta implicación teórica, la política es asumida como un sistema parcial de la sociedad, que opera con funciones específicas, ya que sus decisiones producen efectos vinculantes. De igual manera crea su diferenciación específica con otros sistemas (jurídico, económico, familiar, medicina, entre otros). 
El giro epistémico de la observación de la política en el contexto de la sociedad diferenciada implica cambiar las superposiciones y relaciones de dominantes/dominados y amigos/enemigos por una relación comunicativa mediante el poder. Entonces, se observa la política como un sistema que es parte de la sociedad, cuyo modo de comunicación incrustado es el poder; con el cual — como todo sistema diferenciado y operante a través de códigos funcionales propios- se produce la autorreferencia y la heterorreferencia del sistema político.

De acuerdo con Luhmann (1995), el poder es un medio simbólicamente generalizado de la política, el cual permite conseguir la adaptación de la conducta improbable de ego que se genera en la sociedad. De esta manera, el autor indica que: "La teoría del poder logra una perspectiva general sobre formas de influencia que se tornan accesibles una vez que se trasciende el concepto limitado de poder" (p. 7). Este - como medio simbólicamente generalizado- es una carga de atributos simbólicos de un proceso de influencia para asegurar que las selecciones que hace ego sean las mismas de las premisas de alter.

La consecuencia, el poder y su pérdida se convierten en el foco de la formación de aquel sistema en el que todo medio de ejercicio político (por ejemplo, presión social, protestas, huelgas, mandatos, mecanismos) se convierte en el simbológico generalísimo del control político del poder.

$\mathrm{Al}$ respecto, el poder no está a cargo de un algún o en la reserva de una estructura, sino que está en las operaciones comunicativas de los sistemas sociales y psíquicos, para escoger —con decisión propia- una alternativa con efectos vinculantes. Así, el poder es siempre relacional, pues es un medio de comunicación con el que los sistemas se hilan para reducir o ampliar el poder político.

Con esta perspectiva, ningún sistema político puede garantizar una pérdida total o limitada del poder. Es solo un proceso de cognición de la complejidad y la reducción hecha por los sistemas vinculados comunicativamente por el código de poder y su lado de oposición: no poder; con lo que se elabora la presentación selectiva de esa realidad. Esto no significa que toda la comunicación 
política sea una amenaza abierta o encubierta del poder, lo que sí revela es el ámbito central del sistema político, como las alternativas de sentido y realización que conforman las decisiones y que necesariamente son transmitidas desde un sistema a otro como poder político.

Otra característica de la forma moderna de la política - como sistema social autorreferente- es que, por la atribución de la diferenciación, es un sistema que se componen de subsistemas, como la política, la administración y lo público (Luhmann, 1993, p. 62). Al respecto, Torres (2009) precisa que "en el sistema político este proceso de diferenciación interna se puede reconocer en el surgimiento de un propio orden de cargos". (p. 177); implica también que sus subsistemas, mediante la diferenciación, desempeñan funciones específicas que conducen a la conformación de la unidad de este sistema político.

De acuerdo con lo anterior, cada subsistema del sistema político se diferencia conforme a su autorreferencia de la siguiente manera. En el subsistema político operan las acciones políticas decisivas que configuran la complementariedad de roles entre la administración ${ }^{2}$ y lo público, a lo que Luhmann denomina democracia. Unejemplosonlos partidos políticos, los cuales permiten asentar la comunicación entre el público y la administración. El otro subsistema, la administración, se encuentra ligado al aparato estatal ${ }^{3}$ con sus diferenciaciones, como, por ejemplo, el Gobierno y el cuerpo legislativo, cuyas instituciones

\footnotetext{
2 Con Luhmann (1993) hay que comprender el concepto de administración como la organización institucionalmente dada por el Estado o el Gobierno (Teoría política del estado de bienestar).

3 Luhmann (1993) precisa que la diferenciación interna que se da en el Estado es “[...] según el esquema de división de poderes" (Teoría política del estado de bienestar, p. 63). En consecuencia, el esquema de división de los Estados varía, lo cual abre la circunstancia a que la división del Estado es más amplia conforme a lo que establece la Constitución del Estado. Por ejemplo, de acuerdo con el artículo 113 de la Constitución Política de Colombia, el Estado está dividido en tres poderes: el ejecutivo, que es la administración de gobierno; el legislativo que es creador de las normas; y el judicial, que es la administración de justicia. En cuanto a este último, conforme a lo que indica el artículo 228, la Constitución es un poder que opera conforme al código derecho/no derecho, propio del sistema del derecho. Por ende, hay que diferenciar lo que corresponde a la función política y lo que refiere a la función judicial como parte del sistema del derecho, ya que ello depende de la distinción de la que se parta en la observación del sistema.
} 
generan decisiones vinculantes por mandatos o premisas aportadas por la política. Finalmente, el subsistema público hace referencia a todo aquello que participa de modo decisivo en las acciones políticas y que reacciona frente a las decisiones de la administración; como indica Gonnet (2010), "hace referencia a aquellas organizaciones, actores y/u opinión pública que influyen en los desarrollos del sistema político" (p. 305), los cuales intervienen, a su vez, a través de acciones políticas.

Entonces, la función de cada subsistema es específica y cada uno opera conforme a sus elementos particulares, los cuales son movidos exclusivamente por la política. En consecuencia, todo aquello que tenga que ver con la política establece límites de autorreferente y heterorreferente, que permiten construir la unidad del sistema político diferenciado funcionalmente. Luhmann (1993, p. 54) lo sitúa así: “[...] de forma que todo elemento —en nuestro caso: toda decisión política - remite siempre a esta unidad de construcción, independiente de cualquier otro significado que pueda tener por su contenido o en relación con el entorno".

Ante la dirección comprensiva del sistema político desde la teoría de Luhmann, se desarrolla el aspecto operante de la autorreferencia mediante la clausura operativa. Sin embargo, las condiciones que forman la imposibilidad funcional del sistema se convierten en su propia posibilidad. Esto lleva a la necesaria complementariedad mediante la heterorreferencia, como medio de apertura operativa del sistema respecto a su entorno.

Este flujo conceptual de la teoría de Luhmann sehace más pragmático conforme al contexto de observación del sistema político, el cual aparece en relación con el ser humano por los enlaces comunicativos surtidos en momentos y contingencias determinadas. Un ejemplo de ello es la observación de la relación de la administración pública con el ciudadano en el contexto de Santiago de Chile (Chile) y Pereira (Colombia). En este entorno observacional, la administración pública está en correlación con el sistema psíquico, el que es reconocido como público-ciudadano para designar un tipo de identidad y de actividad política, a pesar de ser categorizado como ciudadano en 
la relación de poder político con la administración pública. Esto le exige a este sistema no reducir a aquél por el rol dado en momento de las actitudes y relaciones al interior del campo político.

Entonces, el ejercicio de observar las relaciones del sistema de administración pública depende del entorno — público de ciudadanos-, lo que hace la mirada compleja - aunque pareciera desordenada - pero válida de formular, porque tanto uno como el otro proveen de suficiente información con las que crean realidad y posibilidad. Miremos las formas operativas de las relaciones administración pública/público ciudadanos en el escenario comparado de la ciudad de Santiago de Chile (Chile) y Pereira (Colombia).

\section{LAS FORMAS DE LA RELACIÓN ADMINISTRACIÓN PÚBLICA/ Público cIUdadanos en El ESCENARIO de SANTIAGo dE Chile (Chile) y Pereira (Colombia)}

Para generar una comprensión sobre las formas de ampliar el sentido del ciudadano, se observa cómo se producen los enlaces comunicativos entre este y la administración pública. En el contexto chileno, en necesario partir del proceso de transformación generado por la movilización social, en torno al grito popular Democracia ya a partir de 1983, que estaba conformado por movimientos sociales, políticos, organizaciones no gubernamentales (ONG), entro otros actores sociales. Esto generó el encuentro entre lo social y lo político en el ciclo de protestas contra la dictadura (Delamaza, 1999).

Este movimiento generó el restablecimiento de la democracia en Chile, en marzo de 1990, con la llegada al poder de Patricio Aylwin, quien fue elegido democráticamente en diciembre de 1989. Con él se iniciaba el proceso y transición de la dictadura a la democracia; sin embargo, como revela Garcés y Valdés (1999), el proceso de transición chileno se desarrolló bajo la constitución definida por los militares y bajo sus reglas, a pesar de haber sido 
derrotados en el plebiscito de 1988. Según los autores, el sistema representativo chileno presenta limitaciones en los procesos de participación ciudadana, más aún, cuando un cuarto del Senado fue designado por diversas autoridades del Estado, con ausencia de las formas y mecanismos democráticos.

El análisis de Garcés y Valdés a la democracia chilena muestra el aseguramiento del Concejo de seguridad Nacional del poder militar sobre el poder civil, en especial cuando se puedan producir momentos de crisis. De esta manera, se creó una prolongación de las fuerzas armadas sobre el mando del país y el jefe de Estado quedó inhibido para la remoción de los mandos militares. Este bosquejo presentado por Garcés y Valdés (1999) llevó a conformar una inferencia radical en el análisis del sistema de Gobierno democrático chileno, que sufrió un estrangulamiento del acceso al parlamento de las minorías y movimientos sociales contrarios al pensamiento de extrema derecha, además del aseguramiento de un tercio de los votos para la conformación del Senado. Por ende, es muy acertado observar que cualquier reforma constitucional no ha contado con una mayoría para ser llevada a cabo.

Esta forma democrática en Chile es denominada gobiernos de la concertación (Garcés y Valdés, 1999; Paredes, 2011; Pressacco y Rivera, 2015), en donde los procesos de participación ciudadana o los movimientos sociales han sido excluidos de los procesos de transición y se afirma la gobernabilidad; es decir, una apuesta a la integración social regulada por la ley de la oferta y la demanda, criterios técnicos, para el gasto social y los propósitos de disciplinamiento y paz social de un Estado central en proceso de descentralización (Garcés y Valdés, 1999, p. 3).

Se identifica como una forma de Gobierno democrático en su transformación política, que le crea al ciudadano una marginación en el ejercicio del poder. Sin embargo, como rescata Paredes (2011), “[...] las fuerzas sociales jugaron un papel trascendental en la lucha por un sistema más democrático, generándose un déficit de participación ciudadana y ciudadanía participativa en los 20 años de gobiernos concertacionistas". (p. 473). Aunque, como acla- 
ran Garcés y Valdés, hay otro lado del análisis académico —en visión de Luhmann, otra información-en donde comparten la limitación de la participación en aras de la gobernabilidad.

Con la llegada de Michel Bachelet a primera mano se instaló una percepción diferente de los gobiernos de la concertación, ya que inició su mandato con la promesa de inclusión y participación de la ciudadanía en la conducción del país, a la cual denominó Gobierno Ciudadano (2006-2010). En voz de Delamaza (2011), se creó una iniciativa de carácter consultivo e informativo, o al menos, buscaba hacerse cargo de la deuda de la concertación con una ciudadanía participativa y el déficit de participación ciudadana. Esta acción constituyó un conformador de expectativa.

Sin embargo, es agudo el análisis de Delamaza (2011) y dePressacco y Rivera (2015) sobre el gobierno desarrollado por Bachelet, pues aclaran que las iniciativas presidenciales nuevamente no han prosperado, pero sí crearon un experimentalismo democrático, en donde las iniciativas desplegadas por la concertación solo siguen una continuidad de pretexto útil (Pressacco y Rivera, 2015, p. 489). Así, el gobierno de la expresidenta Bachelet se puede interpretar como aquél que rediseñó el imaginario institucionalizado de la gobernabilidad hacia la participación de la ciudadanía, en la conquista de la gobernabilidad.

Los autores también demarcan el surgimiento de la desconfianza ciudadana en relación con los referentes del sistema político, como la administración pública o los mecanismos especializados (Luhmann, 1998) para hacer más activa la participación ciudadana. Sin embargo, ante la clausura operativa generada por la administración pública, siempre estarán las selecciones y decisiones del sistema de relación. Es el caso del público de ciudadanos, quienes pueden gestionar, desde su conciencia como sistema, la posibilidad de ampliar la condición (o condiciones) mediante la participación política con que se refrenda el poder político del sistema psíquico.

Un ejemplo de ello es una novedosa iniciativa que se creó a través de internet para generar un espacio de participación del público 
de ciudadano (figura 1). "Tu constitución es el nombre del nuevo sitio web, que fue creado por la fundación del expresidente Lagos - Democracia y Desarrollo - en la cual buscaba fomentar la participación ciudadana" (González, 2015).

Figura 1. Participación ciudadana en nueva Constitución

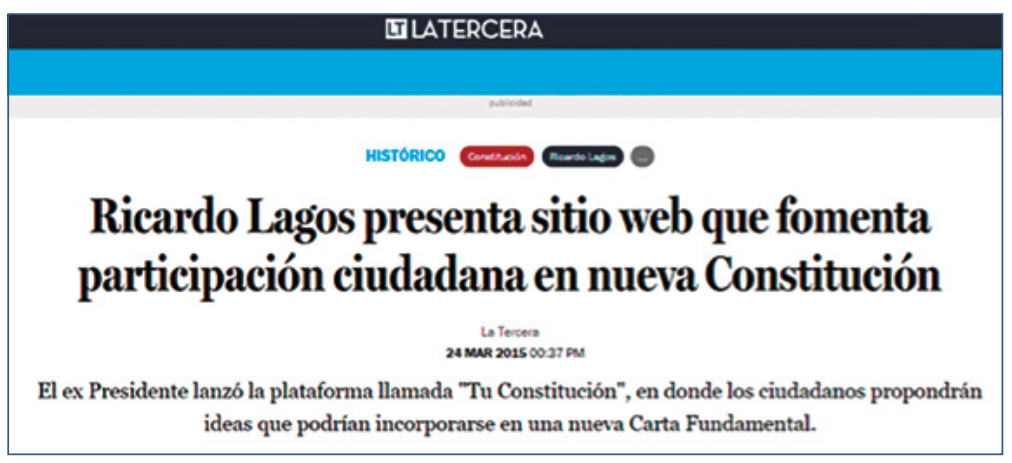

Fuente: González (2015) publicado en el periódico digital La Tercera.

Con esta acción, se halló sentido a la concepción luhmaniana de los mecanismos especializados, pues la participación ciudadana pasa de una realidad física a una realidad virtual, en la búsqueda de escenarios en los que el público de ciudadanos chilenos pueda hacer efectiva la expectativa creada por la administración pública (presidente). Así, las posibles acciones de las personas, que a primera interpretación son una acción individual, surgen de su vivencia por los actuares de una administración que excluye los intereses del ciudadano.

Ahora bien, en el contexto de las unidades político-administrativas $^{4}$ no es abismal la dinámica entre las decisiones y las acciones políticas presentadas, pues en ellas hay una reproducción de las decisiones políticas configuradas desde la clausura operativa de la administración pública de orden central, pese a que Chile es

4 Las comunas corresponden a la división político-administrativa en Chile. 
un Estado territorialmente descentralizado (Constitución Política de la República de Chile, art. 3, 1983). Se puede circunscribir el control de poder político por parte de la administración pública nacional, en cuanto a las decisiones políticas vinculantes bajo el referente de poder político clausurado para los territorios. Santiago de Chile no es la excepción.

En un artículo de prensa de El Mostrador (2018) (figura 2), se destaca que "Solo 6 de los 52 municipios cuenta con una 'unidad de participación ciudadana' en su estructura burocrática. Seguidamente 25 de los 52 municipios tienen un Encargado(a) de participación ciudadana" (Montencinos, 2018).

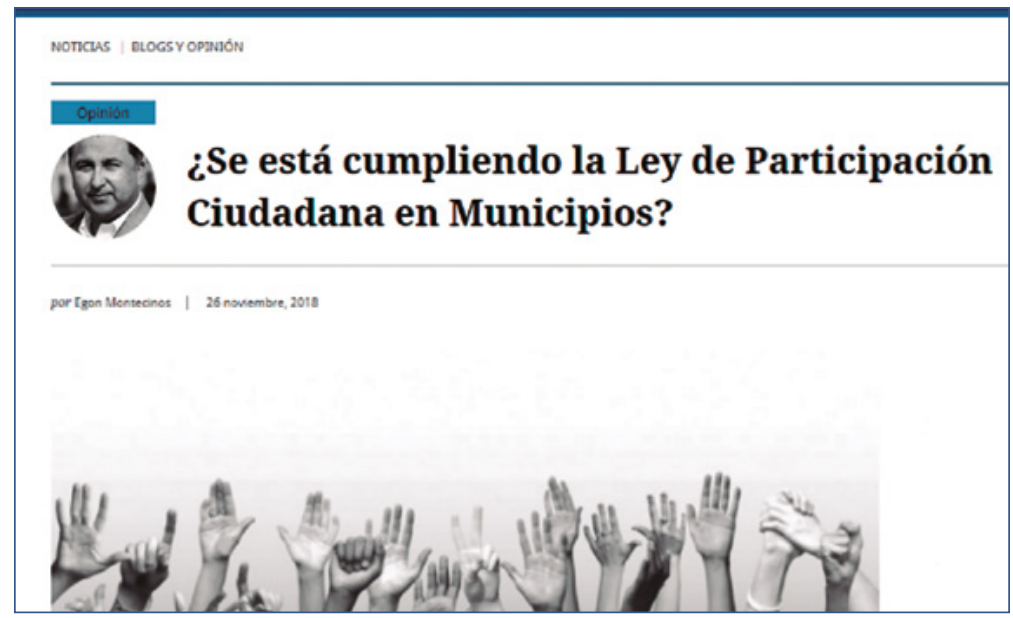

Fuente: Montencinos (2018) publicado en el diario digital El mostrador.

Lo enlazado ante el mecanismo de participación creado por el estamento central para las comunas del territorio nacional sigue constituyendo un cierre del ciudadano como un sistema psíquico en las municipalidades y perpetuando el ocultamiento de la democracia como una unidad emergente de la relación administración pública 
y público de ciudadanos. Esto crea una selectividad en el ciudadano, que converge ante la expectativa de la democracia. Ahora, para el 2020, mediante mecanismos de protesta, el ciudadano orienta las decisiones políticas vinculantes hacia el sistema psíquico, no hacia el económico. En un artículo de El Periódico, titulado Miles de chilenos salen a la Plaza Italia de Santiago en otro viernes de protestas (14 noviembre de 2020) (figura 3), se menciona que:

La apertura de un proceso constituyente era vista como la solución para una gran parte de los manifestantes que desde el 18 de octubre de 2019 protestan contra la desigualdad del país, por considerar que es la vía que permitirá establecer un nuevo modelo de país que desate el corsé neoliberal que ampara la actual Carta Magna, redactada durante la dictadura de Augusto Pinochet (1973-1990). (párrafo 2)

Figura 3. Plebiscito. Nueva Constitución en Chile

\section{|AMBIENTE TENSO \\ Miles de chilenos salen a la Plaza Italia de Santiago en otro viernes de protestas}

Como ya se ha hecho costumbre en las últimas semanas, las manifestaciones sociales continuan luego del plebiscito para redactar una nueva Constitución en Chile

\section{El Peribdico}

SANTIAGO - SÁBADO, 14/11/202:0 - 02:11

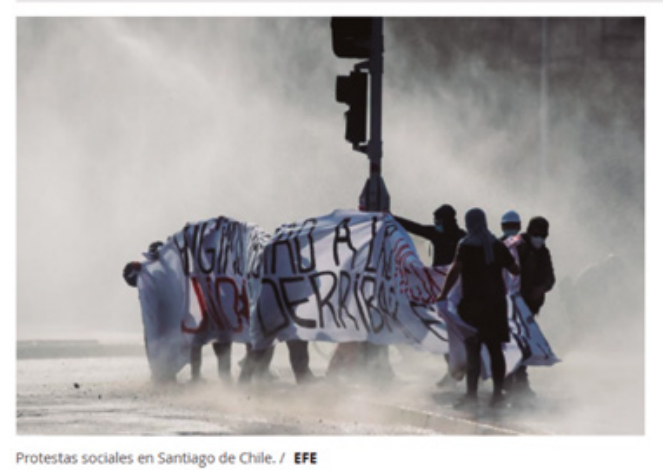

Fuente: El Periódico (2020). 
Así, Santiago de Chile se convirtió en una de las ciudades más representativas para orientar decisiones políticas vinculantes de los ciudadanos, ante las expectativas creadas por la administración pública (central) desde el año 1983. La protesta social se transformó en mecanismo para exigir (poder) y cambios en la manera de configurar la democracia mediante la opacidad del ciudadano como sistema de relación. En actividades registradas por los medios de comunicación chilena (El Mostrador, El Periódico, La Tercera), en las protestas sociales iniciadas desde octubre de 2019 el ciudadano de Santiago de Chile enlazó la decisión del presidente Piñeros de incrementar las tarifas del metro como no vinculante, lo que conllevó a decisiones del público de ciudadanos excluidos de la decisión política encabezada desde 1983 por la administración pública (central). Así lo informó el público de ciudadano.

"Es hermoso, muy bueno y positivo [...] El pueblo de Chile se tiene que unir y tenemos que creer en que nosotros podemos hacer las cosas", dijo a AFP Viviana Donoso, una manifestante de 43 años. [...] "Vengo a celebrar algo que nunca imaginé que iba a pasar en Chile después que recuperamos la democracia", dijo a la AFP Olga Neira, una médica de 68 años que asistió a la manifestación con su hija. (Bloomberg, AFP y EFE, 2020)

De otro lado, en el contexto de Pereira (Colombia) y con el referente de la Constitución Política de 1991, le otorgo al municipio el carácter entidad político-administrativa y ente territorial descentralizado. Sin embargo, la Ley 489 del 29 de diciembre de 1998, en su artículo 7, fijó un límite a la distribución de competencias y responsabilidades en materia social por parte de los municipios, pues normativamente todavía se mantenía la centralización en lo administrativo y financiero ante posibles decisiones provistas por el municipio para decidir sobre recursos económicos y políticos que este pudiera tomar.

Este aspecto - diseñado desde la administración pública (central) - recrea la dualidad implícita en el sistema político de permitir/no permitir ante mecanismos políticos emergentes entre la administración pública (local) y el público de ciudadanos, como 
son las políticas públicas. En el núcleo del artículo 7 de la indicada ley se crea un control de ejecución de la política pública dentro de las entidades territoriales, en especial porque la administración pública de Pereira debe respetar el direccionamiento de la planeación regulada por la Ley 152 del 15 de julio de 1994. En esta ley se crearon los procedimientos y mecanismos para la elaboración, aprobación, ejecución, seguimiento, evaluación y control de los planes de desarrollo en las entidades descentralizadas y, en consecuencia, la asignación presupuestal para el desarrollo de políticas gestada en el municipio.

Así, en materia política, las entidades territoriales — como en el caso específico de las políticas públicas- deben tener la posibilidad de estar determinadas por el Gobierno nacional para que, en la elaboración y adopción del Plan de Desarrollo Municipal, se le aseguren recursos presupuestales desde la nación, para garantizar su ejecución. De lo contrario, los municipios deben de asumir la carga presupuestal para para la ejecución de planes, programas, proyectos producto de una política pública gestada en el municipio.

Con base en lo anterior, es posible comprender la locución de Arroyave Alzate (2011) en cuanto al límite de la participación dentro de las entidades territoriales ante la posibilidad de generarse decisiones públicas como forma de negociación y arreglo mutuo entre actores, pero que ante lo financiero y administrativo son controladas por la nación. Esto permite inferir que las formas de permitir y restringir la asignación de recursos económicos por parte de la nación a las políticas públicas mellan en las entidades descentralizadas la posibilidad de que los alcaldes o concejales propongan la elaboración este tipo de políticas. Por otro lado, el público de ciudadanos tiene la posibilidad de equilibrar la relación de responsabilidad, necesidades y recursos para la formación y ejecución de una política pública.

Por ejemplo, en el desarrollo de la política pública de la cultura de la legalidad institucionalizada mediante el acuerdo municipal 23 de 2010 y el decreto 1097 del 5 de diciembre de 2011, se creó la expectativa cognitiva y normativa de la cultura de la 
legalidad dirigida a los ciudadanos de la municipalidad de Pereira. Lo anterior como tema relevante en el ámbito del sector público, el sector privado y la sociedad civil para lograr justicia y respetar los derechos sociales, el desarrollo de la democracia y los derechos humanos en la medida que: "[...] los ciudadanos lleguen a reconocer su derecho y capacidad para participar en la creación e implementación de las leyes, y que la cultura de la legalidad cambie fundamentalmente la dinámica de las instituciones estatales, haciéndolas más eficientes, eficaces y justas" (Secretaría de Planeación Municipal, 2010, p. 10).

Pero, en el contexto de observación de Pereira, se asumió el ejercicio sistémico por relaciones comunicativas que hilan el ciudadano y la administración pública y que se cierran y se abren entre sí ante el enlace comunicativo de la política pública de la cultura de la legalidad. Esto debido a que el ciudadano tiene la posibilidad -mediante la selección en los ejercicios de comunicación- de ejercer/no ejercer el excedente de posibilidades (vivencia y acción) que la política pública construye, como enlace comunicativo con la administración.

La manera de observar las relaciones comunicativas de apertura y cierre entre cada elemento de observación (ciudadano y administración) se hace desde revisiones llevadas a cabo por la prensa: El Diario (La Tarde) ${ }^{5}$ y el Tiempo, además de la revisión de los informes de seguimiento y desarrollo de la política pública de la cultura de la legalidad editados por el observatorio de políticas públicas de la alcaldía de Pereira. La información y los actos de comunicar que se siguieron de ambos elementos del sistema parten del 2010 hasta el 2020.

En primer lugar, se guarda una distancia en los enlaces comunicativos entre el ciudadano y la administración, ya que en ambos la responsabilidad de cada uno se encuentra presente; es decir,

5 En la actualidad, El Diario es el periódico de circulación de la ciudad de Pereira, el cual compró al periódico La Tarde en el año de 2016, y quedó como la única gaceta para la ciudad. Por lo cual, el lector puede encontrar registros de noticias del periódico La Tarde en relación con los análisis de noticias que se hicieron entre la administración y el ciudadano de Pereira. 
desde la administración se enuncia cuál es la corresponsabilidad del ciudadano y así la responsabilidad de la administración queda relevada. De esta manera se comunica la acción y esta recae sobre el ciudadano.

Esta política pública pretende generar habitantes conscientes y respetuosos, desde una óptica ciudadana cívica con un sentido de pertenencia por la ciudad y sus habitantes, para ello se han enfocado en tres líneas estratégicas, plasmadas en el fortalecimiento institucional, formación para la democracia y la convivencia ciudadana. El cumplimiento de este deber es lo que posibilita a las autoridades competentes actuar de manera efectiva en contra de los actos que causan una grave afectación de los derechos colectivos. (Alcaldía de Pereira, 2019)

Es así como los intereses deben transitar en una relación común entre la administración y el ciudadano, según artículo tercero del acuerdo 23 de 2010, pero para el ciudadano la administración estaría cerrada a la posibilidad de la acción política. Sin embargo, el efecto se revierte cuando el ciudadano se percata de que la acción política de la administración y la expectativa esperada no van a traer consigo una acción política de clausura de la administración.

Mienten, muchos, estructurando banderas que no utilizan. Proponiendo misión y visión que no se realizan. Tarjeta para su militancia es una farsa. Seguimos improvisando y propugnando por alianzas cómicas. A la vuelta, mañana no reclamamos porque somos electores momentáneos, ocasionales, que no exigimos ni aplicamos voto inteligente, a conciencia, con racionalidad. No. Aplicamos aquello del que me da sin vergüenza alguna. (Rodríguez, 2019)

La intención de la comunicación, en el marco de los dos elementos de los sistemas representados anteriormente, evidencia que aún no se encuentran conectados. Esto conlleva a una selección que no simboliza a la política pública de la cultura de la legalidad como una expectación conjunta de ciudadano y administración. En 
segundo lugar, el ciudadano — quien representa la relación directa de satisfacción o de cumplimiento operativo de la política pública de la cultura de la legalidad-siente que aún no se tiene un enlace comunicativo que represente su sentir.

La característica de la conectividad que permite el enlace de los dos sistemas, para el caso del ciudadano y la administración, es la conducta de selectividad en cuanto a las acciones políticas que la administración vincula con el ciudadano. Esto, mediante cifras con las que se le pretende generar al ciudadano una experiencia no propia y, al mismo tiempo, le traduce unas acciones (figura 4).

Figura 4. Seguimiento a la política pública de la cultura de la legalidad

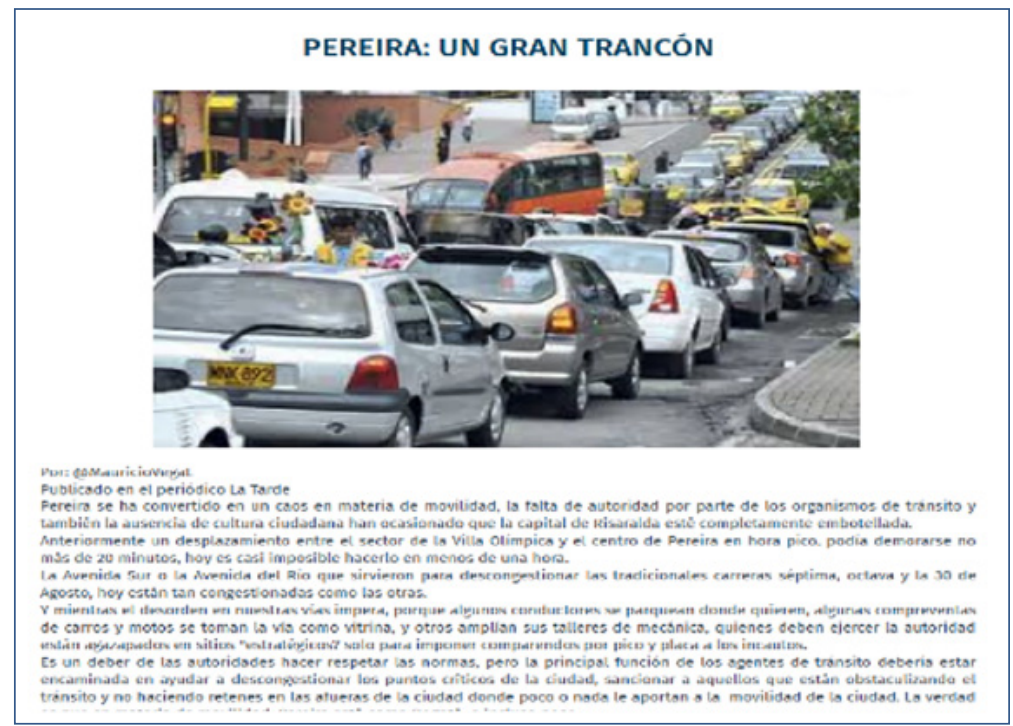

Fuente: Vega (2015).

Sin embargo, la conducta selectiva entre los dos sistemas se presenta en el marco de casi todas las políticas públicas, desde donde el ciudadano empieza a adoptar las dinámicas de los sistemas sociales. Esta relación hace que se contengan, pero igualmente no permite dinamizar la misma política pública, porque no se 
encuentra un circuito comunicativo que permita la interconexión de los intereses entre los dos.

En tercer lugar, la comunicación contenida en el marco de los dos sistemas sociales representa el sentir de la política pública de la cultura de la legalidad. En este caso se puede evidenciar que el ciudadano no siente una representación congruente con su interés. Por ejemplo: "Pereira se ha convertido en un caos en materia de movilidad" (Vega, 2015).

Los medios masivos, como el periódico La Tarde, informan sobre la representación del entorno de la administración (ciudadano) (tabla 1).

Tabla 1. Pereira: divergencia en los logros por estrategías

\begin{tabular}{|c|c|c|c|c|c|c|}
\hline \multirow[t]{2}{*}{ Estrategia } & \multicolumn{5}{|c|}{ Año de seguimiento } & \multirow{2}{*}{$\begin{array}{c}\text { Avance } \\
\text { promedio } \\
(2012-2016)\end{array}$} \\
\hline & 2012 & 2013 & 2014 & 2015 & 2016 & \\
\hline $\begin{array}{l}\text { Movilidad y } \\
\text { espacio público }\end{array}$ & $46 \%$ & $90 \%$ & $89 \%$ & $82 \%$ & $77 \%$ & $77 \%$ \\
\hline $\begin{array}{l}\text { Comunicación } \\
\text { pública }\end{array}$ & $100 \%$ & $100 \%$ & $100 \%$ & $75 \%$ & $100 \%$ & $95 \%$ \\
\hline Educación & $54 \%$ & $100 \%$ & $94 \%$ & $53 \%$ & $76 \%$ & $76 \%$ \\
\hline $\begin{array}{l}\text { Medio ambiente } \\
\text { y desarrollo } \\
\text { sostenible }\end{array}$ & $100 \%$ & $83 \%$ & $86 \%$ & $89 \%$ & $73 \%$ & $86 \%$ \\
\hline $\begin{array}{l}\text { Derechos huma- } \\
\text { nos y seguridad } \\
\text { ciudadana }\end{array}$ & $100 \%$ & $100 \%$ & $93 \%$ & $60 \%$ & $100 \%$ & $91 \%$ \\
\hline
\end{tabular}

Fuente: Dirección de Planeación Estratégica e Integración Regional (s. f.).

En la noticia citada se evidencia que la información se envía a alter, en este caso la administración pública que no responde al interés expresado por el ego. En este sentido se presenta una clausura operativa, ya que no hay un enganche entre el alter y el ego. Por ejemplo: 
[...] “'La Plaza de Bolívar es intocable, eso lo sabemos todos!”, exclamó un experto en el tema y agregó una pregunta: “Si le ordenaron (a la dirección de espacio público) reubicar ese puesto, ¿Por qué lo hizo hacia la Plaza de Bolívar y no fuera de ella?".

[...] “El Estado no puede ir a patearlo (al vendedor), tiene que garantizar la dignidad humana, el debido proceso, la confianza legitima, hacer una notificación y después de esta verificar la documentación y trasladar el proceso a un inspector de Policía para que le haga un comparendo, aplique el Código de Policía", explicó. (¿Pereira perdió la pelea por el espacio público?, 2017)

Esta serie de expresiones caracterizan los enlaces comunicativos del ciudadano con respecto a la representación de la política pública de la cultura de la legalidad. Se puede evidenciar que estos dos elementos de los sistemas — que hacen parte de los sistemas sociales- no están simbolizando las acciones de los demás en el marco de la puesta en marcha de esta política pública. Esto deja una posibilidad comunicativa en potencialidad, más no en actualidad, entre los dos elementos del sistema. La intención de la política pública abre la expectación en la medida de la posibilidad de la comprensión que se pueda generar de uno, del otro o en una relación igual como unidades independientes, pero como unidad política de la cultura de la legalidad.

$\mathrm{Al}$ parecer, el enlace que provee la política pública de la cultura de la legalidad en relación con el ciudadano se está cerrando a la administración; además, porque no se encuentran evidencias de manifestaciones que establezcan una relación entre los dos sistemas. A pesar de estar conectados y teniendo intereses similares, parece que no se logra una comunicación que enlace los intereses de la política en los dos elementos de relación.

Como se mencionó, los procesos comunicativos entre los sistemas expuestos a través del alter — la administración - y el ego —el ciudadano - no están conectados para buscar que la política pública de la cultura de la legalidad respondiera a los intereses de los dos sistemas y se desarrollara una comunicación selectiva, en 
la que se evidencie que los mensajes del alter y de ego generan un enganche que permita la inclusión de elementos del mensaje para la interpretación de los sistemas, que luego desarrollen de manera operativa las acciones que involucra la política de cultura de la legalidad en la ciudad de Pereira.

Así las cosas, se establecen relaciones de poder ejercidas desde las dinámicas de la administración y que son representadas a través de las acciones, pero conectadas desde el mensaje. Se nota como el alter y el ego entran en tensión por el manejo de intereses específicos al interior de la administración pública local, aunque el ciudadano sea reconocido en la expectativa la política pública de la cultura de la legalidad. Es decir, que al no presentase un enlace comunicativo entre el diseño y la ejecución (actualidad) de la política pública de la cultura de la legalidad, en la relación del sistema y el entorno se crean clausuras/aperturas.

\section{Conclusiones: ampliación/no aplicación del CIUDADANO, UN PROBLEMA FUNDADO EN LA EXCLUSIÓN/ INCLUSIÓN}

A primera vista, el análisis de relación sistémica entre la administración pública y el sistema psíquico, comprendido como públicos ciudadanos en el escenario de la ciudad de Santiago de Chile (Chile) y Pereira (Colombia) serían muy distantes. Pero gracias a la teoría de los sistemas sociales como medio epistémico de análisis es posible encontrar trazos comunicativos muy similares de ambos contextos. Esto permite comprender las dinámicas de interpenetración de ambos sistemas desde la figura de la autorreferencia y la heterorreferencia que se produce entre ambos, sin importar la relación comunicativa que se establezca, ya sea abierta, selectiva o incluso de clausura/apertura operativa.

La complejidad de relaciones entre administración pública y público de ciudadanos gozan del valor contingente de la relación, en el que el enganche comunicativo no necesariamente está direccionado a las mismas pretensiones políticas. Lo interesante es 
que, en momentos determinados y bajo la dimensión temporal, el público de ciudadanos se clausura creando medios de oposición a la instalada por la administración pública nacional, o local, y creando una turbulencia a la seguridad del control del poder establecida. Por ejemplo, el resultado de la desmedida protección del modelo económico que ahoga la autorreferencia del ciudadano.

Si se piensa en la relación administración pública y público ciudadano, es importante precisar que no tiene un orden condicionado de superior a inferior, entendido como que la sociedad es una relación entre sistema/entorno, por lo cual, las construcciones políticas en la sociedad se hacen desde una permanente relación de poder y no poder. Para los dos casos, el público de ciudadanos es codificado por medios de fuerza hacia el poder político de la administración pública, lo cual se evidencia en el cierre comunicativo del ciudadano como sistema de conciencia autónoma. Esto deriva en el ocultamiento del ser humano en un contexto de relaciones políticas y, en consecuencia, de la formación de la democracia como unidad de la relación administración pública/ciudadanos.

\section{REFERENCIAS}

Alcaldía Municipal de Pereira. (2011, 5 de diciembre). Decreto 1097 de 2011. Por medio del cual se reglamenta el Acuerdo No 23 de 2010, por el cual se adopta como política pública el subprograma: "cultura de la legalidad".

Alcaldía de Pereira. (2019, 15 de julio). Política pública de la cultura de la legalidad, un compromiso que todos debemos conocer. Sala de prensa. https://bit. ly/3lamYU9

Arroyave Alzate, S. (2011). Las políticas públicas en Colombia. Insuficiencias y desafíos. Forum. Revista Departamento de Ciencia Política, 1(1), 95-111. https: / / bit.ly/30DoBk1

Bedoya, O., Jaramillo, C. y Castiblanco, A. (2004). Paradoja de la ciudadanía en Pereira: entre los ciudadanos jurídicos los itinerantes (2000-2003). Editorial Magisterio

Concejo Municipal de Pereira. (2010, 9 de junio). Acuerdo 23 de 2010. Por medio del cual: se establece los lineamientos generales para la política pública de cultura de la legalidad para el municipio de Pereira. https: / / bit.ly / 3rG0i0J

Congreso de la República de Colombia. (1994, 19 de julio). Ley 152 de 1994. Por la cual se establece la Ley Orgánica del Plan de Desarrollo. Diario Oficial 41450. https: / / bit.ly/3t9t8XA 
Congreso de la República de Colombia. (1998, 30 de diciembre). Ley 489 de 1998. Por la cual se dictan normas sobre la organización y funcionamiento de las entidades del orden nacional, se expiden las disposiciones, principios y reglas generales para el ejercicio de las atribuciones previstas en los numerales 15 y $16 \mathrm{del}$ artículo 189 de la Constitución Política y se dictan. otras disposiciones. Diario Oficial 43 464. https: / / bit.ly / 38xmNNQ

Delamaza, G. (1999). Movimientos Sociales en la Democracia de Chile. En P. Drake e I. Jaksic (eds.), El modelo chileno: Democracia y desarrollo en los noventa (pp. 377-405). Lom Ediciones.

Delamaza, G. (2011). Espacio público y participación ciudadana en la gestión pública en Chile: Límites y posibilidades. Polis (Santiago), 10(30), 45-75. https: / / doi.org/10.4067/S0718-65682011000300003

Durkheim, E. (1982). Las formas elementales de la vida religiosa. Akal editor

Garcés, M. y Valdés, A. (1999). Estado del Arte de la Participación Ciudadana en Chile. documento preliminar para OXFAM-GB. https: / / bit.ly/2PRnw5T

Gonnet, J. P. (2010). La política desde la teoría de sistemas sociales de Niklas Luhmann. Argumentos (México, D.F.), 23(64), 303-310.

González, K. (2015, 24 de marzo). Ricardo Lagos presenta sitio web que fomenta la participación ciudadana en la nueva constitución. Periódico digital La Tercera. https: / / bit.ly/2OKCGJE

Rodríguez, A. (2019). Las redes: ¡los nuevos directorios políticos! Periódico Eje noticias. https: / / bit.ly /312fE40

Luhmann, N. (1993). Teoría política del estado de bienestar. Alianza editorial.

Luhmann, N. (1995). Poder. Universidad Iberoamericana-Anthropos

Luhmann, N. (1998). Sistemas sociales: lineamientos para una teoría general. Anthropos Editorial del Hombre.

Luhmann, N. (2007). La sociedad de la sociedad. Herder y Universidad Iberoamericana.

Miles de chilenos salen a la Plaza Italia de Santiago en otro viernes de protestas. (2020, 14 de noviembre). El Periódico. http:/ / bit.ly/3cO9ry8

Montecinos, E. (2018, 26 de noviembre). Se está cumpliendo la ley de participación ciudadana. El mostrador. https: / / bit.ly/3cld6mJ

Montenegro, S. (2009, 15 de marzo). ¿Sociedad estática o sociedad dinámica? El Espectador. https:/ / bit.ly/30A2P0t

Mosquera Osorio, J. (2017). Informe de seguimiento a la política pública de cultura de la legalidad año 2016. Secretaría de Planeación Municipal. Alcaldía de Pereira. https: / / bit.ly/3rEwhOV

Paredes, P. J. (2011). Ciudadanía, Participación y Democracia. Deuda y Déficit en los 20 años de "Democracia" en Chile. Polis. Revista Latinoamericana, 10(28), 473-499. https: / / doi.org/10.4067/S0718-65682011000100022

¿Pereira perdió la pelea por el espacio público? (2017, 19 de agosto). El Tiempo. https: / / bit.ly / 2OIItPV

Pressacco, C. y Rivera, S. (2015). Democracia, participación y espacio local en Chile. Papel Político, 20(1), 9-33. 
Joaquín Andrés Gallego Marín, César Augusto Castaño, Erika María Bedoya - Ampliación

del ciudadano desde un enfoque sistémico en Pereira (Colombia) y Santiago de Chile (Chile)

Simmel, G. (2002). Sobre la individualidad y las formas sociales. Universidad Nacional de Quilmes.

Secretaría de Planeación Municipal. (2010). Política Pública en: Cultura de la Legalidad. Alcaldía de Pereira.

Torres N, J. (2009). Niklas Luhmann. La política como sistema social. Universidad iberoamericana, ITESCO.

Ulrich, B. (1998). La sociedad del riesgo: hacia una nueva modernidad. Paidos.

Vega, M. (2015). Pereira: un gran trancón. Cámara de Comercio de Pereira, La Tarde. http: / / bit.ly/3f1UI55

Zapata-Barrero, R. (2001). Ciudadanía, democracia y pluralismo cultural: hacia un nuevo contrato social. Anthropos Editorial. 